\title{
Static and dynamic routing under disjoint dominant extreme demands
}

\author{
A. Frangioni ${ }^{\mathrm{a}}$, F. Pascalic,*, M.G. Scutellà ${ }^{\mathrm{b}}$ \\ ${ }^{a}$ Polo Universitario della Spezia, Via dei Colli 90, 19121 La Spezia, Italy \\ ${ }^{b}$ Dipartimento di Informatica, Università di Pisa, Largo B. Pontecorvo 3, 56127 Pisa, Italy \\ ${ }^{c}$ Department of Computer Science, Universitè Libre de Bruxelles, Boulevard du Triomphe CP 210/01, 1050 Bruxelles, \\ Belgium
}

\begin{abstract}
This paper considers the special case of the robust network design problem where the dominant extreme points of the demand polyhedron have a disjoint support. In this case static and dynamic routing lead to the same optimal solution, both for the splittable and the unspittable case. As a consequence, the robust network design problem with (splittable) dynamic routing is polynomially solvable, whereas it is co $\mathcal{N} \mathcal{P}$-Hard in the general case. This result applies to particular instances of the single-source Hose model.
\end{abstract}

Keywords: Robust Optimization, Network Design, Hose model

\section{Introduction}

Let $\mathcal{G}=(\mathcal{V}, \mathcal{A})$ be a directed network, with $|\mathcal{V}|=$ $n$ and $|\mathcal{A}|=m$. Let $\mathcal{K}$ be a set of $k$ origin-destination pairs which represent users that wish to communicate, and $c_{i j}$ denote the non-negative cost of installing a unit of capacity along $(i, j) \in \mathcal{A}$. Let $\mathcal{D}$ be a bounded non-empty polyhedron describing the possible non-simultaneous demands between the pairs in $\mathcal{K}$. The robust network design problem (RND) on $\mathcal{G}$ consists of determining a minimum cost capacity allocation for the $\operatorname{arcs}$ of $\mathcal{G}$ such that the network is able to support each demand in $\mathcal{D}$.

Several variants and generalizations of RND have been proposed in the literature in the last decade. Concerning the routing constraints, each pair may be required to communicate through a single path (unsplittable routing), or the traffic can be split among different paths (splittable routing). Moreover, the routing can be dynamic, that is, it can change as the demand varies in $\mathcal{D}$, or static, that is, the same routing template must be used for each demand in $\mathcal{D}$. Observe that static routing - also referred to as "oblivious" [1] or "stable" [3] — can be preferable in network applications where migrating from one routing to another one is costly [2]. Clearly, even if the objective function only depends on the capacity allocation costs, the optimal solution value depends on the routing constraints. In general, splittable routing leads to a cheaper solution than unsplittable routing. RND with unsplittable routing is

\footnotetext{
* Corresponding author

Email addresses: frangio@di.unipi.it (A. Frangioni), pascali@di.unipi.it (F. Pascali), scut@di.unipi.it (M.G. Scutellà)
}

in fact a generalization of the directed Steiner tree problem, whereas RND with splittable routing provides a fractional relaxation, and the gap between the optimal values of the two problems is known to be $\Omega\left(\log ^{2} n /(\log \log n)^{2}\right)$ [12]. Similarly, dynamic routing leads to cheaper solutions than static routing; for instance, [8] shows that the gap between the costs of dynamic and static routing is $\Omega(\log n)$ for a family of instances of RND.

Concerning the shape of the demand polyhedron $\mathcal{D}$, the most widely studied case in the literature is the so-called Hose model, which simply specifies a bound on the maximum total traffic that each node can receive (considering the destination nodes) or send out (for the origins). The particular case in which a single node can send traffic along the network, while all the other nodes are potential receivers, is referred to as the single-source Hose model.

From a time complexity perspective, the very special case in which $\mathcal{D}$ is a singleton (the so-called nominal case) is clearly polynomially solvable both in the unsplittable and in the splittable scenario, as it can be easily solved by computing shortest paths between the origin-destination pairs. The splittable static case is still polynomially solvable for any separable convex $\mathcal{D}$ using the standard ellipsoid argument [10], in particular, compact linear programming formulations can be devised when $\mathcal{D}$ is a polyhedral set $[1,13]$. On the other hand, RND is $\mathcal{N} \mathcal{P}$ Hard in the unsplittable routing model (both static and dynamic) $[4,11]$. RND is also difficult in the splittable dynamic case, as it is coN $\mathcal{N}$-Hard even in the special case of the single-source Hose model both in directed [11] and in undirected networks [5]. Thus, dynamic routing is, in general, substantially 
more difficult than static routing. This has motivated the study of "intermediate scenarios" such as the one where the demands in $\mathcal{D}$ can be served by two alternative routing templates [16], which allows one to obtain cheaper solutions w.r.t. static routing while being computationally tractable in some cases. Another line of approach is to study special cases of RND that are solvable in polynomial time due to the special structure of the demand polyhedron $\mathcal{D}$. Specifically, in the case of symmetric Hose polyhedra on undirected networks, RND with static unsplittable routing is polynomially solvable [9]. Similarly, when $\mathcal{D}$ is built upon a discrete number of scenarios whose number is polynomial in $n$ and $m$, then RND with splittable dynamic routing is polynomially solvable since a compact linear programming formulation can be devised [14]. The fact that the time complexity of the problem depends on $\mathcal{D}$ has motivated approaches [15] where the demand polyhedron can be reduced by discarding some dominated demand vectors. Indeed, it is useful to remark that in the nominal case (i.e., when $\mathcal{D}$ is a singleton) the problem is polynomially solvable because static and dynamic routing lead to the same optimal solution. For a more detailed survey on complexity results concerning RND, its variants and its generalizations, the interested reader is referred to [4] where several interesting variants of RND are discussed, such as cases where the optimum routing support is required to be a tree, and where the objective function involves congestion aspects.

In this paper, we partially answer to one of the open questions in [4]: in what cases RND with splittable dynamic routing can be solved in polynomial time? We prove that this is true if $\mathcal{D}$ is a disjoint dominant extreme demand polyhedron. This is for instance the case of the single-source Hose model with unitary bounds, or when the source bound is smaller than any receiver bound, or still when the source bound is greater than or equal to the sum of all receiver bounds. As in the nominal case, the result follows from the stronger property that imposing a static or a dynamic routing leads to the same capacity solution, and this is true both for the splittable and the unsplittable case. Although all results are proved for the case of directed networks, they easily generalize to the undirected case.

The paper is organized as follows. In Section 2 we recall some basic results and notions about RND. Then we introduce the new definition of disjoint dominant extreme demand polyhedron and prove the main result. In Section 3 we study the application of the obtained results to the single-source Hose model.

\section{The disjoint extreme demand polyhedron}

Let $y$ denote a vector of routing variables, i.e., $y_{i j}^{s t}$ be the fraction of the demand of $(s, t) \in \mathcal{K}$ to be routed along the $\operatorname{arc}(i, j) \in \mathcal{A}$. Then $y: \mathcal{A} \times \mathcal{K} \rightarrow$ $[0,1]$ is a routing template if it satisfies the usual flow conservation constraints:

$\sum_{(j, i) \in B S(i)} y_{j i}^{s t}-\sum_{(i, j) \in F S(i)} y_{i j}^{s t}=\phi_{i}^{s t}=\left\{\begin{aligned}-1 & \text { if } i=s \\ 1 & \text { if } i=t \\ 0 & \text { otherwise. }\end{aligned}\right.$

for all $i \in \mathcal{V}$ and $(s, t) \in \mathcal{K}$, where, as customary, $F S(i)$ and $B S(i)$ denote the set of arcs leaving node $i$ and entering it, respectively. This can be equivalently restated in compact form as $E y^{s t}=\phi^{s t}$ for all $(s, t) \in \mathcal{K}$, where $E$ denotes the node-arc incidence matrix of $\mathcal{G}$ and $\phi^{s t}=\left[\phi_{i}^{s t}\right]_{i \in \mathcal{V}}$. Hereafter we shall denote the set of all routing templates by $\mathcal{Y}$, and by $x=\left[x_{i j}\right]_{(i, j) \in \mathcal{A}}$ a vector of design variables.

Definition 1. Given a routing template $y \in \mathcal{Y}$ and a capacity allocation $x \in \mathbb{R}_{+}^{m}$, the pair $(y, x)$ supports $\mathcal{D}$ if

$$
\sum_{(s, t) \in \mathcal{K}} y_{i j}^{s t} d_{s t} \leq x_{i j} \quad(i, j) \in \mathcal{A}, d \in \mathcal{D} .
$$

In this case, $y$ is said to be a feasible static routing with respect to $x$ and $\mathcal{D}$.

When the routing can change dynamically with $d \in \mathcal{D}, y$ is rather a routing function $y: \mathcal{A} \times \mathcal{K} \times \mathcal{D} \rightarrow$ $[0,1]$, where $y(d)_{i j}^{s t}$ denotes the fraction of the demand of $(s, t)$ to be routed along $(i, j)$ when the demand vector is $d$. Of course, the notion of feasibility has to be changed accordingly.

Definition 2. The capacity allocation $x \in \mathbb{R}_{+}^{m}$ supports $\mathcal{D}$ if, for each $d \in \mathcal{D}$, there exists a routing template $y(d) \in \mathcal{Y}$ such that

$$
\sum_{(s, t) \in \mathcal{K}} y(d)_{i j}^{s t} d_{s t} \leq x_{i j} \quad(i, j) \in \mathcal{A} .
$$

The family $y(d), d \in \mathcal{D}$, is said to be a feasible dynamic routing with respect to $x$.

Of course, (1) implies (2), since one can use the same $y$ for all $d \in \mathcal{D}$, while the converse, in general, is not true. Hence, dynamic routing may allow to accommodate cheaper capacity allocations than static routing. In fact, let $M P R$ and $S P R$ denote, respectively, splittable (multi path) and unsplittable (single path) static routing scheme, while $F R$ and $I R$ denote respectively splittable and unspittable dynamic routing scheme. Moreover, let $P^{*}$ denote the optimal value of a problem RND when the routing scheme $P$ is adopted. We can then extend a result of [13] to the dynamic setting, by comparing the solution value of instances with the same underlying data (network $\mathcal{G}$ and demand polyhedron $\mathcal{D}$ ). 
Theorem 1. Both in the directed and in the undirected case,

$$
F R^{*} \leq M P R^{*} \leq S P R^{*}
$$

Proof. The first inequality has already been commented upon: any static routing is a dynamic one. The second inequality comes from the fact that $M P R$ is the relaxation of $S P R$ obtained by removing the requirement to use a single path for each origindestination pair.

By the same token, $I R^{*} \leq S P R^{*}$; however, nothing is known in general about $M P R^{*}$ and $I R^{*}$.

An instrumental concept to our analysis is that of domination among demand vectors [15]. A demand vector $d^{1}$ dominates $d^{2}$ if any capacity allocation $x: \mathcal{A} \rightarrow \mathbb{R}_{+}^{m}$ supporting $d^{1}$ also supports $d^{2}$. Moreover, $d^{1}$ totally dominates $d^{2}$ if any pair $(y, x)$ supporting $d^{1}$ also supports $d^{2}$. Clearly, total domination implies domination. A nice characterization of total domination is the following:

Theorem 2. [15, Theorem 2.5] If $d_{s t}^{1} \geq d_{s t}^{2}$ for all $(s, t) \in \mathcal{K}$, then $d^{1}$ totally dominates $d^{2} ;$ the converse implication holds true if $\mathcal{G}$ is a complete graph.

Recall that the anti-dominant of a set $S \subseteq \mathbb{R}^{m}$ is the set $A(S)=\left\{x \in \mathbb{R}^{m}: \exists y \in S: x \leq y\right\}$, where the inequality is intended to hold componentwise. The next lemma states that, given a routing template for each of a discrete set of demand vectors, it is possible to build up (in linear time) a feasible routing template for each demand vector belonging to the anti-dominant of the convex hull of the given demand vectors.

Lemma 1. Any capacity allocation $x \in \mathbb{R}_{+}^{m}$ that supports a finite set $\mathcal{D}=\left\{d^{h} \in \mathbb{R}^{k}\right\}_{h \in H}$ of demand vectors also supports any demand $d$ in the anti-dominant of $\operatorname{conv}(\mathcal{D})$.

Proof. We will show that the capacity vector $x$ supports any demand vector in $\operatorname{conv}(\mathcal{D})$. This is sufficient, since Theorem 2 allows one to immediately extend the result to the anti-dominant of $\operatorname{conv}(\mathcal{D})$. In fact, each demand in the anti-dominant of $\operatorname{conv}(\mathcal{D})$ is totally dominated by at least one demand in $\operatorname{conv}(\mathcal{D})$. By hypothesis, for each $h \in H$ there exists a routing template $y\left(d^{h}\right)$ which is feasible w.r.t. $x$ and $d^{h}$. Consider a vector of convex multipliers $\lambda \in \Lambda^{|H|}$, where $\Lambda^{p}=\left\{\lambda \geq 0: \sum_{h} \lambda_{h}=1\right\}$ is the unitary simplex in dimension $p$. Now consider the corresponding demand vector $d^{\lambda}=\sum_{h \in H} \lambda_{h} d^{h} \in$ $\operatorname{conv}(\mathcal{D})$. We claim that

$$
z\left(d^{\lambda}\right)_{i j}^{s t}=\sum_{h \in H} \lambda_{h}\left(y\left(d^{h}\right)_{i j}^{s t} d_{s t}^{h} / d_{s t}^{\lambda}\right)
$$

is a feasible routing template w.r.t. $x$ and $d^{\lambda}$.
Of course, (3) is well-defined only if $d_{s t}^{\lambda}>0$. However, if $d_{s t}^{\lambda}=0$ then any routing template relative to the pair $(s, t)$ supports it. Therefore, w.l.o.g. we shall restrict our attention to the case $d_{s t}^{\lambda}>0$.

Now, for all $v \in \mathcal{V}$ and $(s, t) \in \mathcal{K}$

$$
\begin{aligned}
& \sum_{(i, j) \in \mathcal{A}} E_{i j}^{v} z\left(d^{\lambda}\right)_{i j}^{s t}= \\
= & \sum_{(i, j) \in \mathcal{A}} E_{i j}^{v}\left(\sum_{h \in H} \lambda_{h} y\left(d^{h}\right)_{i j}^{s t} d_{s t}^{h} / d_{s t}^{\lambda}\right)= \\
= & \left(1 / d_{s t}^{\lambda}\right) \sum_{h \in H} \lambda_{h} d_{s t}^{h} \sum_{(i, j) \in \mathcal{A}} E_{i j}^{v} y\left(d^{h}\right)_{i j}^{s t}= \\
= & \left(1 / d_{s t}^{\lambda}\right) \sum_{h \in H} \lambda_{h} d_{s t}^{h} \phi_{v}^{s t}=\phi_{v}^{s t},
\end{aligned}
$$

where (4) is due to algebraic manipulations and (5) follows by the hypothesis that $y\left(d^{h}\right)$ are routing templates for all $h \in H$. Moreover, for each $(i, j) \in \mathcal{A}$

$$
\begin{aligned}
& \sum_{(s, t) \in \mathcal{K}} d_{s t}^{\lambda} z\left(d^{\lambda}\right)_{i j}^{s t}= \\
= & \sum_{(s, t) \in \mathcal{K}} d_{s t}^{\lambda}\left(\sum_{h \in H} \lambda_{h} y\left(d^{h}\right)_{i j}^{s t} d_{s t}^{h} / d_{s t}^{\lambda}\right)= \\
= & \sum_{h \in H} \lambda_{h} \sum_{(s, t) \in \mathcal{K}} y\left(d^{h}\right)_{i j}^{s t} d_{s t}^{h} \leq \sum_{h \in H} \lambda_{h} x_{i j}=x_{i j}
\end{aligned}
$$

where (6) follows from (3) and (7) follows by algebraic manipulations and the hypothesis that $y\left(d^{h}\right)$ is feasible w.r.t. $x$ and $d^{h}$ for each $h \in H$ (as well as by the definition of $\lambda$ ).

Now, let $\mathcal{R}$ be the set of the extreme points of the anti-dominant of a bounded and non-empty polyhedron $\mathcal{D}$, and $I(d)=\left\{h: d_{h}>0\right\}$ denote the support of vector $d$. We define the following:

Definition 3. A demand polyhedron $\mathcal{D}$ is disjoint dominant extreme demand if

$$
I(r) \bigcap I(s)=\emptyset
$$

for each pair $(r, s) \in \mathcal{R} \times \mathcal{R}$ with $r \neq s$. We will denote this kind of polyhedron by $\mathcal{D}_{D d e}$.

Note that the number of the dominant extreme points of $\mathcal{D}_{D d e}$ is bounded from above by $k$, since it is not possible to partition the set $\mathcal{K}$ in more than $k$ subsets which satisfy condition (8). We are now ready to state the main result.

Theorem 3. The following statements

$$
\begin{array}{r}
x \text { supports } \mathcal{D}_{D d e} \\
\exists z \in[0,1]^{m \times k}:(x, z) \text { supports } \mathcal{D}_{D d e}
\end{array}
$$

are equivalent. 
Proof. $(10) \Rightarrow(9)$ is trivial: as already remarked, a static routing is also dynamic. To prove $(9) \Rightarrow(10)$ observe that, if $x$ supports $\mathcal{D}_{D d e}$, then it supports each dominant extreme point of $\mathcal{D}_{D d e}$, say $r \in \mathcal{R}$. That is to say, a routing template $y(r)$ exists which is feasible w.r.t. $x$ and the associated demand vector $d^{r}$ for each $r \in \mathcal{R}$. Thus, from Lemma 1 , the routing template $z(d)$ determined according to (3) supports each $d^{\lambda}$ in the anti-dominant of $\mathcal{D}_{D d e}$ :

$$
z\left(d^{\lambda}\right)_{i j}^{s t}=\sum_{r \in \mathcal{R}} \lambda_{r}\left(y\left(d^{r}\right)_{i j}^{s t} d_{s t}^{r} / \sum_{r \in \mathcal{R}} \lambda_{r} d_{s t}^{r}\right) .
$$

Let $r(s t)$ denote the - unique, by condition (8)extreme demand point such that $d_{s t}^{r(s t)}>0$, by observing that, if $d_{s t}^{r}=0$ for each $r \in \mathcal{R}$, then the origin-destination pair $(s, t)$ can be disregarded, since its demand is always null. We get

$$
z\left(d^{\lambda}\right)_{i j}^{s t}=\lambda_{r(s t)}\left(y\left(d^{r(s t)}\right)_{i j}^{s t} d_{s t}^{r(s t)} / \lambda_{r(s t)} d_{s t}^{r(s t)}\right) .
$$

That is, for all $(s, t) \in \mathcal{K}$

$$
z\left(d^{\lambda}\right)_{i j}^{s t}=y\left(d^{r(s t)}\right)_{i j}^{s t} .
$$

Therefore, actually $z\left(d^{\lambda}\right)$ is independent on $d^{\lambda}$. If we denote it by $z$, we get that $z$ is a static routing template which, by the construction within the proof of Lemma 1, is indeed feasible w.r.t. $x$ for all $d \in$ $\mathcal{D}_{D d e}$. Hence, $(x, z)$ supports $\mathcal{D}_{D d e}$.

Corollary 1. If the routing templates corresponding to the dominant extreme points of $\mathcal{D}_{D \text { de }}$ are integer valued, i.e., $y\left(d^{r}\right) \in\{0,1\}^{m \times k}$ for each $r \in \mathcal{R}$, then the static routing template $z$ in Theorem 3 is unspittable too, that is, $z \in\{0,1\}^{m \times k}$.

Proof. Just consider formula (11): if $y\left(d^{r}\right)$ are integer valued for all $r \in \mathcal{R}$, then $z$ is integer, too.

Theorem 3 and Corollary 1 thus allow to strengthen the results of Theorem 1 under special circumstances. In fact, when the hypothesis of Theorem 3 holds true one has:

$$
F R^{*}=M P R^{*} \leq I R^{*}=S P R^{*}
$$

Observe that the obtained result is stronger than the one implied by the fact that $\mathcal{D}_{D d e}$ has a polynomial number of dominant extreme points. In fact, such a property just assures that, in the case of dynamic routing, RND on $\mathcal{D}_{D d e}$ is solvable in polynomial time, since a compact linear programming formulation can be devised, while Theorem 3 and Corollary 1 show a stronger connection (i.e. same optimal solution) between static and dynamic routing, both in the splittable and in the unsplittable case. It is an open question as to whether some classes of polyhedra do exist which do not have a polynomial number of dominant demand vectors, but such that dynamic and static routing lead to the same optimal solution. The obtained results will be applied to some relevant Hose cases in the following section.

\section{The single-source Hose model}

Consider the so-called asymmetric Hose polyhedron $[6,7], \mathcal{D}_{\text {Asym }}=\left\{d \in \mathbb{R}_{+}^{k}: \sum_{t:(v, t) \in \mathcal{K}} d_{v t} \leq\right.$ $\left.b_{v}^{\text {out }}, \sum_{s:(s, v) \in \mathcal{K}} d_{s v} \leq b_{v}^{\text {in }}, v \in \mathcal{V}\right\}$, where each terminal $v \in \mathcal{V}$ has an upper bound $b_{v}^{\text {out }}$ on the cumulative amount of traffic that can be sent by $v$, as well as an upper bound $b_{v}^{i n}$ on the cumulative amount of traffic that can be received by $v$. If the traffic can be sent by a single source node, say $r$, whereas only destination nodes $t \in \mathcal{T} \subseteq \mathcal{V} \backslash\{r\}$ can receive traffic, one obtains the single-source Hose polyhedron $\mathcal{D}_{S s}=$ $\left\{d \in \mathbb{R}_{+}^{|\mathcal{T}|}: \sum_{t \in \mathcal{T}} d_{r t} \leq b_{r}^{\text {out }}, d_{r t} \leq b_{t}^{\text {in }} t \in \mathcal{T}\right\}$. In this special case the source $r$ belongs to all pairs, thus the number of the commodities is equal to the number of destination nodes (i.e., $k=|\mathcal{T}|$ ).

\subsection{Case of unit bounds}

Let us first consider the specialization of the singlesource Hose model where all bounds are unitary, i.e., the unitary single-source Hose polyhedron $\mathcal{D}_{U s s}=$ $\left\{d \in \mathbb{R}_{+}^{k}: \sum_{t \in \mathcal{T}} d_{r t} \leq 1\right\}$. Clearly, the inequalities $d_{r t} \leq 1$ relative to the destination nodes $t \in \mathcal{T}$ are redundant and can be dropped. $\mathcal{D}_{U s s}$ is therefore a very simple polyhedron, i.e. the $k$-dimensional tetrahedron $\Delta^{k}$. It is well-known that its extreme points are 0 and the unitary vectors $e^{t}, t \in \mathcal{T}$, of the orthogonal basis of the demand space; in other words, $\Delta^{k}=\operatorname{conv}\left(0, \Lambda^{k}\right)$. Since these dominant extreme points have a disjoint support, then $\Delta^{k}$ is a disjoint dominant extreme demand polyhedron and Theorem 3 thus applies. It follows that RND with (splittable) dynamic routing is polynomially solvable under the $\mathcal{D}_{U s s}$ model. In addition, static and dynamic routing lead to the same optimal solution, both for the splittable and the unspittable case.

\subsection{Case of unlimited source bound}

When the source node has an unlimited bound $b_{r}^{\text {out }}$ (equivalently, $r$ can push an amount of traffic greater than or equal to the overall requirement of the destination nodes), then there exists a unique dominant demand vector in $\mathcal{D}_{S s}$, which is determined by the destination node bounds. This is the vector, say $d^{b}$, such that $d_{r t}^{b}=b_{t}^{i n}, t \in \mathcal{T}$. The following property holds true:

Theorem 4. If $\sum_{t \in \mathcal{T}} b_{t}^{\text {in }} \leq b_{r}^{\text {out }}$, then $R N D$ under $\mathcal{D}_{S s}$ is equivalent to $R N D$ under $\mathcal{D}=\left\{d^{b}\right\}$, i.e., the nominal case in which all destinations require the maximum amount they can. 
Proof. Since $d_{r t} \leq b_{i n}^{t}$ for all $t \in \mathcal{T}$, one has

$\sum_{t \in \mathcal{T}} d_{r t} \leq \sum_{t \in \mathcal{T}} b_{i n}^{t} \Rightarrow \sum_{t \in \mathcal{T}} d_{r t} \leq b_{r}^{\text {out }} \quad \forall d \in \mathcal{D}_{S s}$

Therefore, the Hose constraint related to the source $r$ is redundant. From Theorem 2 each demand in $\mathcal{D}_{S s}$ is thus totally dominated by the single demand $d^{b}$, which belongs to $\mathcal{D}_{S s}$. The thesis follows.

As a consequence, the single-source Hose model with unlimited source bound can be solved by solving a nominal network design problem w.r.t. the single demand vector $d^{b}$. Therefore, also in this case $F R^{*}=M P R^{*}$, and this is true also in the unsplittable scenario.

\subsection{Source bound limited by each receiver bound}

Consider now the special case of $\mathcal{D}_{S s}$ where $b_{r}^{\text {out }} \leq$ $b_{t}^{i n}, t \in \mathcal{T}$. This case can be reduced to the singlesource Hose model with unitary bounds. Therefore, the same results proved for $\mathcal{D}_{U s s}$ apply. To show this, we need a technical result from [13], where the triplet $(\mathcal{G}, b, c)$ denotes an instance of RND, under the Hose model, for a network $\mathcal{G}$, an upper bound vector $b$ and a cost vector $c$.

Theorem 5. [13, Lemma 3.4] For each $\beta \in \mathbb{R}_{+}$, the instance $(\mathcal{G}, \beta b, c)$ has a feasible solution of value $\beta C$ if and only if the instance $(\mathcal{G}, b, c)$ has a feasible solution of value $C$.

Theorem 6. If $b_{r}^{\text {out }} \leq b_{t}^{\text {in }}$ for all $t \in \mathcal{T}$, then $R N D$ under $\mathcal{D}_{S s}$ is equivalent to $R N D$ under $\mathcal{D}_{\text {Uss }}$.

Proof. Consider an instance of RND with upper bound vector $b=\left(b_{r}^{\text {out }}, b_{1}^{\text {in }}, \ldots, b_{k}^{\text {in }}\right)$. From Theorem 5 we can scale $b$ by a factor $b_{r}^{\text {out }}$, thereby obtaining an equivalent RND problem (up to the chosen scale factor) with upper bound vector $\left(1, \bar{b}_{1}^{i n}, \ldots, \bar{b}_{k}^{i n}\right)$, where $\bar{b}_{i}^{\text {in }}=b_{i}^{\text {in }} / b_{r}^{\text {out }} \geq 1$. Clearly, each inequality $d_{r t} \leq$ $\bar{b}_{i}^{i n}$ can be removed since it is redundant $\left(\sum_{t \in \mathcal{T}} d_{r t} \leq\right.$ $\left.1 \Rightarrow d_{r t} \leq 1\right)$. Therefore, this case reduces to the one discussed in Section 3.1.

\section{Acknowledgement}

We are grateful to the anonymous referee and to the Area Editor, whose precious suggestions helped us to considerably improve the quality of the paper.

[1] D. Applegate and E. Cohen. Making intra-domain routing robust to changing and uncertain traffic demands: understanding fundamental tradeoffs. In Proc. of $S I G$ COMM '03, pages 313-324, NY, USA, 2003. ACM.

[2] W. Ben-Ameur and H. Kerivin. Networks new economical virtual private. ACM Commun., 46(6):69-73, 2003.

[3] W. Ben-Ameur and H. Kerivin. Routing of uncertain traffic demands. Optimization and Engineering, 6(3):283-313, 2005.
[4] C. Chekuri. Routing and network design with robustness to changing or uncertain traffic demands. SIGACT News, 38(3):106-129, 2007.

[5] C. Chekuri, F. B. Shepherd, G. Oriolo, and M. G. Scutellà. Hardness of robust network design. Networks, 50(1):50-54, 2007.

[6] N. G. Duffield, P. Goyal, A. Greenberg, P. Mishra, K. K. Ramakrishnan, and J. E. van der Merive. A flexible model for resource management in virtual private networks. In Proc. of SIGCOMM '99, pages 95-108, NY, USA, 1999. ACM.

[7] J. A. Fingerhut, S. Suri, and J. S. Turner. Designing least-cost nonblocking broadband networks. J. Algorithms, 24(2):287-309, 1997.

[8] N. Goyal, N. Olver, and F. Shepherd. Dynamic vs. oblivious routing in network design. In A. Fiat and P. Sanders, editors, Algorithms - ESA 2009, volume 5757 of Lecture Notes in Computer Science, pages 277-288. Springer Berlin Heidelberg, 2009.

[9] N. Goyal, N. Olver, and F. B. Shepherd. The VPN conjecture is true. In Proc. of STOC '08, pages 443-450, NY, USA, 2008. ACM.

[10] M. Grötschel, L. Lovász, and A. Schrijver. The ellipsoid method and its consequences in combinatorial optimization. Combinatorica, 1:169-197, 1981.

[11] A. Gupta, J. Kleinberg, A. Kumar, R. Rastogi, and B. Yener. Provisioning a virtual private network: a network design problem for multicommodity flow. In Proc. of STOC '01, pages 389-398, NY, USA, 2001. ACM.

[12] E. Halperin, G. Kortsarz, R. Krauthgamer, A. Srinivasan, and N. Wang. Integrality ratio for group steiner trees and directed steiner trees. SIAM J. Comput., 36(5):1494-1511, 2006.

[13] C. A. J. Hurkens, J. C. M. Keijsper, and L. Stougie. Virtual private network design: A proof of the tree routing conjecture on ring networks. SIAM J. of Discr. Math., 21(2):482-503, 2007.

[14] M. Labbé, M. Séguin, P. Soriano, and C. Wynants. Network synthesis with non-simultaneous multicommodity flow requirements. Technical report, ULB, 1999.

[15] G. Oriolo. Domination between traffic matrices. Mathemathics of Operation Research, 33(1):91-96, 2008.

[16] M. G. Scutellà. On improving optimal oblivious routing. Operations Research Letters, 37(3):197-200, 2009. 